\title{
CNTNAP3 Associated ATG16L1 Expression and Crohn's Disease
}

\author{
Yu Qi Qiao, ,2,3,4 Mei Lan Huang, ${ }^{1,2,3,4}$ Qing Zheng, ${ }^{1,2,3,4}$ Tian Rong Wang, ${ }^{1,2,3,4}$ \\ An Tao Xu, ${ }^{1,2,3,4}$ Yuan Cao, ${ }^{1,2,3,4}$ Di Zhao, ${ }^{1,2,3,4}$ Zhi Hua Ran, ${ }^{1,2,3,4}$ and Jun Shen ${ }^{1,2,3,4}$ \\ ${ }^{1}$ Division of Gastroenterology and Hepatology, Renji Hospital, School of Medicine, Shanghai Jiao Tong University, \\ Shanghai 200127, China \\ ${ }^{2}$ Shanghai Institute of Digestive Disease, Shanghai 200127, China \\ ${ }^{3}$ Shanghai Inflammatory Bowel Disease Research Center, Shanghai 200127, China \\ ${ }^{4}$ Key Laboratory of Gastroenterology \& Hepatology, Ministry of Health (Shanghai Jiao Tong University), Shanghai 200127, China
}

Correspondence should be addressed to Zhi Hua Ran; zhihuaran@vip.126.com and Jun Shen; shenjun79@sina.cn

Received 21 November 2014; Revised 17 March 2015; Accepted 17 March 2015

Academic Editor: Chiara De Luca

Copyright (C) 2015 Yu Qi Qiao et al. This is an open access article distributed under the Creative Commons Attribution License, which permits unrestricted use, distribution, and reproduction in any medium, provided the original work is properly cited.

\begin{abstract}
Autophagy is a common physiological process in cell homeostasis and regulation. Autophagy-related gene mutations and autophagy disorders are important in Crohn's disease (CD). The nucleotide oligomerization domain 2-autophagy genes autophagy 16-like 1 (NOD2-ATG16L1) signaling axis disorder contributes to the dysfunction of autophagy. This paper is focused on the relationship between contactin associated protein-like 3 (CNTNAP3) and ATG16L1 expression in Crohn's disease. The results indicated that the expression of ATG16L1 is higher in some CD patients compared to normal controls. ATG16L1 was well correlated with the C-reactive protein (CRP) in some CD patients. In vitro study revealed that CNTNAP3 could upregulate the expression of ATG16L1 and increase autophagy vacuoles.
\end{abstract}

\section{Introduction}

Autophagy, a common mechanism in cell homeostasis and degradation, has emerged to play a critical role in Crohn's disease (CD). Genome-wide association studies (GWAS) revealed that polymorphisms in autophagy-associated genes such as autophagy-related 16-like 1 (ATG16L1) and immunityrelated GTPase family M (IRGM) were risk loci for CD $[1,2]$. Autophagy shows the inhibitory role in inflammasome activation, as indicated by the evidence that downregulation of ATG16L1 leads to increased interleukin- (IL-) $1 \beta$ expression in a mouse model of CD [3]. A cohort study suggested that ATG16L1 loci variant displayed increased levels of proinflammatory cytokine IL-1 $\beta$ and IL-6 in humans [4]. Another study indicated that pro-IL-1 $\beta$ could be specifically sequestered into autophagosomes in macrophages stimulated by toll-like receptor ligands [5]. However, polymorphisms in nucleotide oligomerization domain 2 (NOD2) remain the most prominent genetic risk factor among $\mathrm{CD}$-associated risk loci identified so far $[6,7]$. In recent years, several studies have linked the CD-associated NOD2 mutations to autophagy appearance via the interaction of NOD2 with ATG16L1 $[8,9]$. NOD2-mediated autophagy is needed in bacterial recognition in the dendritic cells. Besides, both CDassociated NOD2 and ATG16L1 mutative CD patients are defective in autophagy induction, bacterial trafficking, and antigen presentation [8]. During bacterial invasion, NOD2 and ATG16L1 will interact together at bacterial entry sites, initiating the collection of the ATG16-ATG5-ATG12 complex to the autophagosomes. However, mutant $\mathrm{CD}$-associated NOD2 (L1007fsinsC) failed to recruit ATG16L1 to the plasma membrane. Consequently, intracellular bacterial degradation by autophagosomes wrapping is defective [9]. This evidence links NOD2 and autophagy-related genes to innate immune responses against bacterial invasion, showing that the NOD2ATG16L1 signaling axis disorder contributes to the deficiency of autophagy in CD [10].

Studies have highlighted that autophagy plays the critical role in maintaining intestinal homeostasis, and dysfunction of autophagy seems to be a risk factor in the onset of chronic intestinal inflammation. Some genetic risk polymorphisms have been found related to autophagy. However, 
most of the autophagy-related polymorphisms were found in specific region or ethnicity. ATG16L1 polymorphism cannot be confirmed as one of the susceptibility loci in $C D$ patients from Asian countries [11, 12]. The selective effects on the cell biology and specialized regulatory properties of ATG16L1 or autophagy in CD patients from Asia are still unclear. Contactin associated protein-like 3 (CNTNAP3) is a gene located in chromosome 9p13.1. Protein encoded by CNTNAP3 gene is a member of NCP family (NeuroxinIV/CNTNAP/Paranodin) of cell-recognition molecules, a distinct subgroup of the neurexins which mediates neuronglial interactions [13]. The function of CNTNAP3 has not been fully detected. Due to our preliminary data, CNTNAP3 expression was found upregulated in the intestinal tissue of the patients with CD. However, it is not known how CNTNAP3 contributes to ATG16L1 or autophagy in intestinal biology or $\mathrm{CD}$ pathogenesis. Thus, in this paper we try to figure out if CNTNAP3 participates in the process of autophagy or ATG16L1 pathway.

\section{Materials and Methods}

2.1. Subjects and Samples. A total of fifteen patients with active $\mathrm{CD}$ and fifteen healthy controls (HC) were enrolled in this study from September 2010 to February 2015. The present research was approved by the Research Ethics Committee of Renji Hospital, School of Medicine, Shanghai Jiao Tong University. Written informed consents were obtained from all subjects before recruitment. Patients were diagnosed based on the clinical manifestations, endoscopy, and pathology, confirmed by two gastroenterologists. All patients were newly diagnosed-no medication history. Patients with infectious diseases, pregnancy, or malignancy were excluded. Colonic biopsy specimens were obtained from all subjects. Tissue samples were obtained from inflamed segments of colons in patients and regions without pathological changes in HC. At the same day of colonoscopy, patients provided a fasting blood sample for measurement of serum C-reactive protein (CRP) and erythrocyte sedimentation rate (ESR). CRP and ESR were performed by routine laboratory tests.

2.2. Cell Culture and Transfection. HeLa cell line and SW620 cell line were obtained from the American Type Culture Collection (ATCC) and maintained in the Shanghai Institute of Digestive Disease. Both HeLa and SW620 cells were grown in Dulbecco's Modified Eagle Medium (DMEM) (Gibco) with $10 \%$ iron-supplemented calf serum (Hyclone), streptomycin $(50 \mu \mathrm{g} / \mathrm{mL})$ (Invitrogen), and penicillin $(50 \mathrm{U} / \mathrm{mL})$ (Invitrogen) at $37^{\circ} \mathrm{C}$ with $5 \% \mathrm{CO}_{2}$ supplement.

To downregulate the CNTNAP3 expression, small interfering (si)RNA oligos were purchased from GenePharma. The siRNA sequences were designed using GenePharma siRNA design center (http://www.genepharma .com/). These siRNA oligopairs were Cntnap3-homo-2039 ( $5^{\prime}$-CGUCUGGGCUUUACUAUAUTT- $3^{\prime}$ and $5^{\prime}$-AUAUAGUAAAGCCCAGACGTT-3') and Cntnap3-homo-1311 ( $5^{\prime}$ GGAAAUGUGUCCUUCUCAUTT- $3^{\prime}$ and $5^{\prime}$-AUGAGAAGGACACAUUUCCTT- $3^{\prime}$ ). The negative control pairs were $5^{\prime}$-UUCUCCGAACGUGUCACGUTT- $3^{\prime}$ and $5^{\prime}$-ACGUGACACGUUCGGAGAATT- $3^{\prime}$. The CNTNAP 3 cDNA was amplified and inserted into pcDNA 3.1 Vector (Invitrogen) to establish pcDNA 3.1-CNTNAP3 for overexpression purpose. The plasmids were amplified in One Shot TOP10F' Chemically Competent Cells (Invitrogen) following the manufacturer's guide and purified with PureLink HQ Mini Plasmid Purification Kit (Invitrogen). CNTNAP3 siRNAs or pcDNA 3.1-CNTNAP3 were transfected into HeLa cells, respectively, with Lipofectamine 2000 Transfection Reagent (Invitrogen).

2.3. RNA Extraction, Reverse Transcription, and RealTime PCR. Total RNA from biopsy specimens and cells was extracted using Cell Culture and Tissue Total RNA Extraction and Preparation Mini Kit (SLNco) according to the manufacturer's instruction. The quantity and quality of RNA were confirmed with a NanoDrop 1000 (NanoDrop, Thermo Scientific-Waltham). The primers were designed using Primer Premier 5.0 software and synthesized from Generay Biotech Co., Ltd: CNTNAP3 (forward 5'-TCGCCACCCAAGGAGGATAT- ${ }^{\prime}$, reverse $5^{\prime}$-TCAAAGGGAGGCTGGAGTCTGT- ${ }^{\prime}$ ); ATG16L1 (forward $5^{\prime}$-AGGACAGGGAGATGCAGATGA- $3^{\prime}$, reverse $5^{\prime}$-GATTGGCTTCCTGGGCTTT- $3^{\prime}$ ); $\beta$-actin (forward $5^{\prime}$-GTCTTCCCCTCCATCGTG$3^{\prime}$, reverse $5^{\prime}$-AGGGTGAGGATGCCTCTCTT- $3^{\prime}$ ). For gene-specific reverse transcription, first strand synthesis was performed with PrimeScript RT reagent Kit (TaKaRa). Quantitative real-time PCRs were conducted on a StepOne Plus device (Applied Biosystems) with SYBR Premix Ex Taq kit (TaKaRa). Data were analyzed by $2-\Delta \Delta \mathrm{Ct}$ algorithm [14].

2.4. Western Blot. Cell lysates were prepared using RIPA buffer (Sigma) containing protease inhibitors (Roche), subsequently agitated on ice for 30 minutes. Pierce BCA Protein Assay Kit (Pierce) was used to measure the protein concentration. Protein electrophoresis was performed with Mini-PROTEA III (Bio-Rad). In 10\% polyacrylamide gels (Tris/glycine), proteins were separated and transferred onto a polyvinylidene fluoride membrane (Bio-Rad). Primary and secondary antibodies were labeled subsequently. Antibodies were applied against CNTNAP3 (rabbit polyclonal antiCNTNAP3, 1:1000, Sigma-Aldrich), ATG16L1 (rabbit polyclonal anti-ATG16L1, 1:1000, Abcam), and GAPDH (rabbit polyclonal anti-GAPDH; 1:2500, Abcam). Goat anti-rabbit IgG-HRP secondary antibody was purchased from Santa Cruz. Experiments were performed in triplicate.

2.5. Autophagy Observation with Fluorescent Microscope. Autophagic vacuoles with monodansylcadaverine (MDC) were stained and assessed as previously described [15]. HeLa and SW620 cells seeded in six- or twelve-well plates were transfected with pSELECT-GFP-LC3 (Invitrogen) using Lipofectamine reagent (Invitrogen) following the manufacturer's instructions. Typically, $1 \times 10^{6}$ cells/mL well in sixwell plates was transfected with $0.5 \mu \mathrm{g}$ plasmids. Transient transfections with cDNAs were performed with Lipofectamine 2000 to label autophagic vacuoles with GFP-LC3 

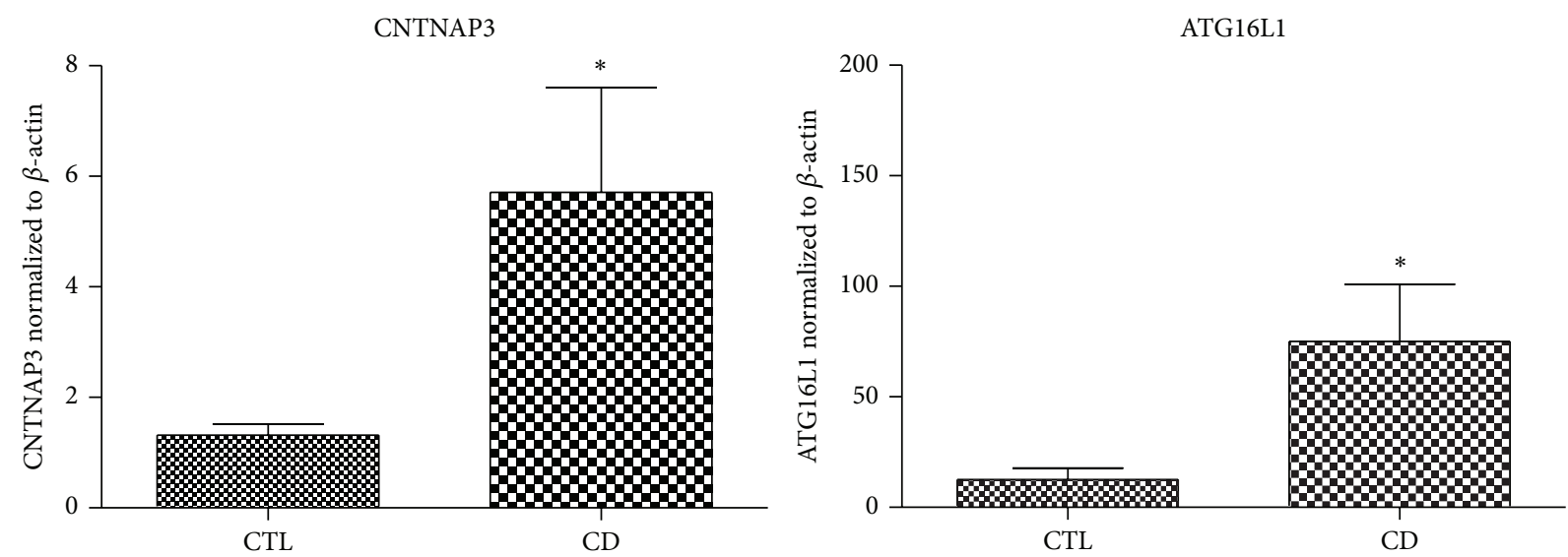

Figure 1: The mRNA expression of CNTNAP3 and ATG16L1 in Crohn's disease. qRT-PCR showed that the levels of both CNTNAP3 $(P=$ $0.029)$ and ATG16L1 $(P=0.024)$ were significantly high in CD patients compared to those in healthy controls.

plasmid for 24 to 72 hours [16]. Cells were fixed with $4 \%$ paraformaldehyde (PFA) in PBS after the treatments. Images were obtained using a fluorescent microscope (GFP filterEx470/40VS495, Em515/30; Zeiss) and GFP-LC3 punctuated dots were determined for triplicates. Quantification of the average number of GFP-LC3 punctuated dots per cell was performed as previously mentioned [17].

2.6. Statistics. GraphPad Prism 5.0 for Windows (GraphPad Software) was used in statistics. $P<0.05$ was considered to be significant with either ANOVA analysis or $t$-test.

\section{Results}

3.1. Characteristics of Included Subjects. A total of fifteen CD patients and fifteen healthy controls were enrolled in the present experiment. After we included the CD patients and HCs, we found that CD patients showed a trend of lower body mass index (BMI). The characteristics of included subjects are listed in Table 1.

3.2. CNTNAP3 and ATG16L1 mRNA Expression Increase in CD Patients Compared with HCs. Expression of CNTNAP3 and ATG16L1 in colonic tissue from CD patients and HCs were detected by qRT-PCR, showing that both CNTNAP3 $(P=0.029)$ and ATG16L1 $(P=0.024)$ were highly expressed in CD patients compared to those in HCs (Figure 1).

3.3. ATG16L1 Correlates with C-Reactive Protein (CRP) Levels in $C D$ Patients. Interestingly, spearman correlation analysis indicated that ATG16L1 and CNTNAP3 mRNA expressions were significantly associated with the serum levels of CRP $(r=0.6238$ for ATG16L1, $P=0.013)(r=0.5711$ for CNTNAP3, $P=0.026$ ) (Figures 2(a) and 2(b)). CNTNAP3 also significantly expressed correlation with the ATG16L1 transcriptomic level $(r=0.8631, P<0.001)$ (Figure 2(c)). Unfortunately, it was found that neither CNTNAP3 $(P=$ $0.273)$ nor ATG16L1 $(P=0.231)$ was significantly correlated with ESR (Figures 2(d) and 2(e)).
TABLE 1: Characteristic of included CD patients and HCs.

\begin{tabular}{lccc}
\hline & $\mathrm{CD}(n=15)$ & $\mathrm{HC}(n=15)$ & $P$ value \\
\hline Age $(\mathrm{yrs})$ & $30.27 \pm 2.074$ & $29.00 \pm 1.604$ & 0.6327 \\
BMI $\left(\mathrm{kg} / \mathrm{m}^{2}\right)$ & $20.82 \pm 0.3448$ & $23.98 \pm 0.3927$ & $<0.0001$ \\
Gender $($ female/male $)$ & $6 / 9$ & $8 / 7$ & \\
Smoking & $2 / 13$ & N/A & \\
Extent & & & \\
$\quad$ Ileocolitis & 12 & N/A & \\
$\quad$ Colitis & 3 & N/A & \\
\hline
\end{tabular}

CD: Crohn's disease; HC: healthy control; N/A: not applicable.

3.4. CNTNAP3 Manipulates the Expression of ATG16L1 in $\mathrm{HeL} a$ and SW620 Cells. Given the evidence that CNTNAP3 elevated expression was closely associated with ATG16L1, we subsequently changed the expression pattern of CNTNAP3 by knocking down and overexpression, to determine the downstream target, namely, ATG16L1 in HeLa and SW620 cells.

Seventy-two hours after transfection, the level of CNTNAP3 was measured by qRT-PCR (Figures 3(a) and 3(b)) and validated with western blot (Figures 3(c) and 3(d)). Compared with cells transfected with empty vectors, CNTNAP3 was significantly higher in CNTNAP3 overexpression cells (HeLa, $P=0.001$ and SW620, $P=0.014$ ) and lower in CNTNAP3 knockdown cells (HeLa, $P=0.013$ and SW620, $P=0.002)$. There was no significant difference between cells transfected with empty vectors and normal controls (HeLa, $P=1.000$ and SW620, $P=0.794$ ).

Results showed that ATG16L1 was lower in CNTNAP3 knockdown cells than that in cells transfected with empty vectors (HeLa, $P=0.013$ and SW620, $P=0.005$ ) (Figures 3(a) and 3(b)). On the other hand, ATG16L1 was significantly higher in cells transfected with CNTNAP3 overexpression plasmid than that in cells transfected with empty vectors (HeLa, $P=0.001$ and SW620, $P=0.052$ ) (Figures 3(a) and $3(\mathrm{~b}))$. There was no significant difference between cells 
ATG16L1

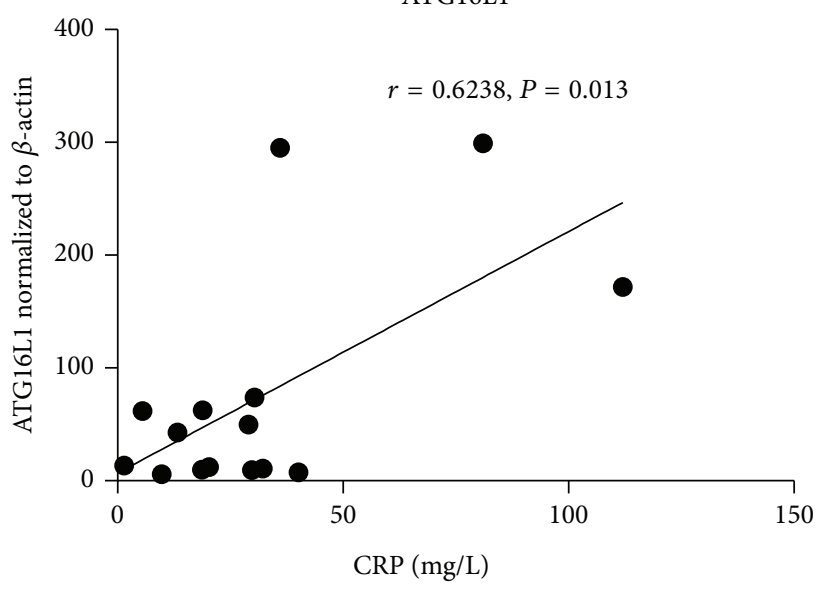

(a)

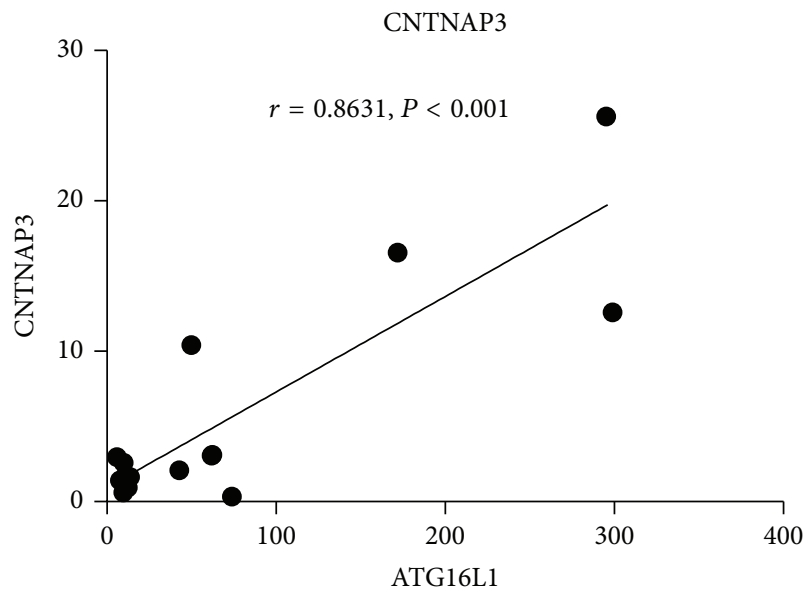

(c)

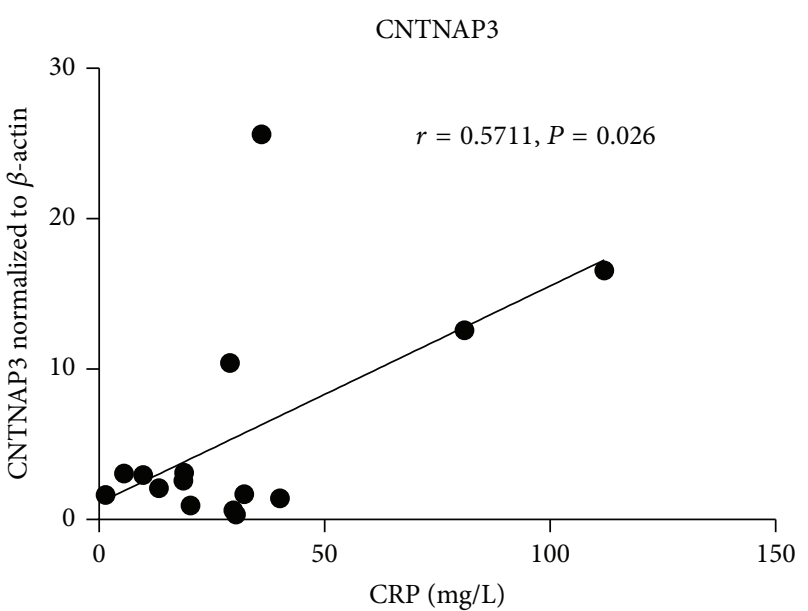

(b)

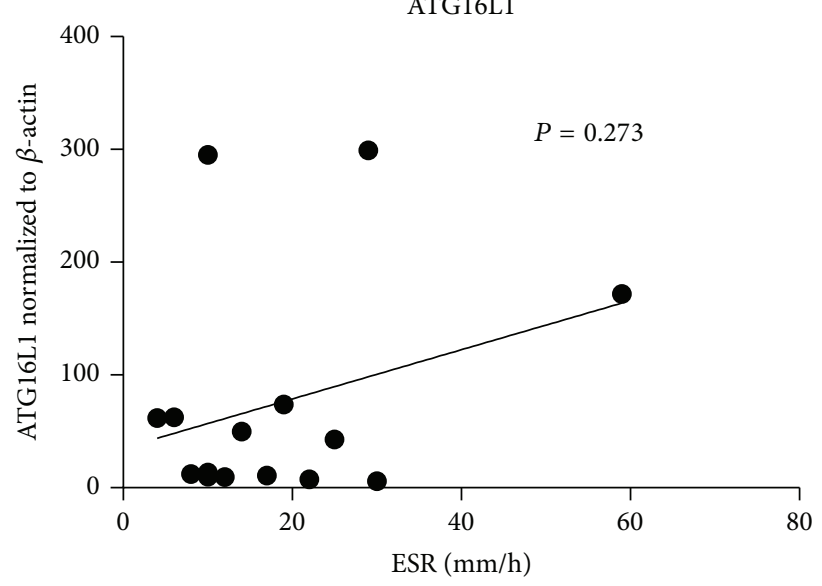

(d)

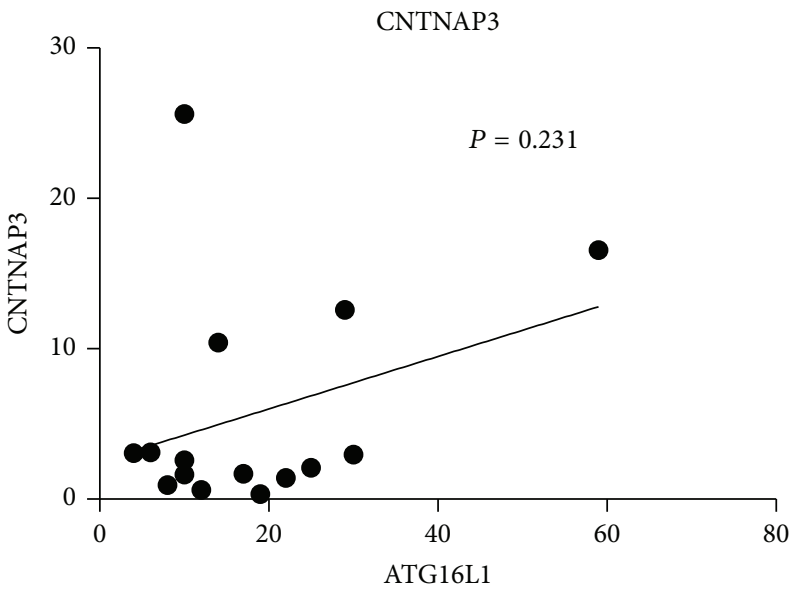

(e)

FIGURE 2: The relationships between CNTNAP3, ATG16L1, and some serum markers. Spearman correlation analysis indicated ATG16L1 mRNA expressions were significantly associated with the serum levels of CRP $(r=0.6238$ and $P=0.013)$ (a). CNTNAP3 was also showed significance correlated with CRP $(r=0.5711$ and $P=0.026)(\mathrm{b})$. CNTNAP3 changes positively correlated with the ATG16L1 level $(r=0.8631$ and $P<0.001)(\mathrm{c})$. Unfortunately, it was found that neither CNTNAP3 $(P=0.273)$ nor ATG16L1 $(P=0.231)$ was significantly correlated with $\operatorname{ESR}(d, e)$. 

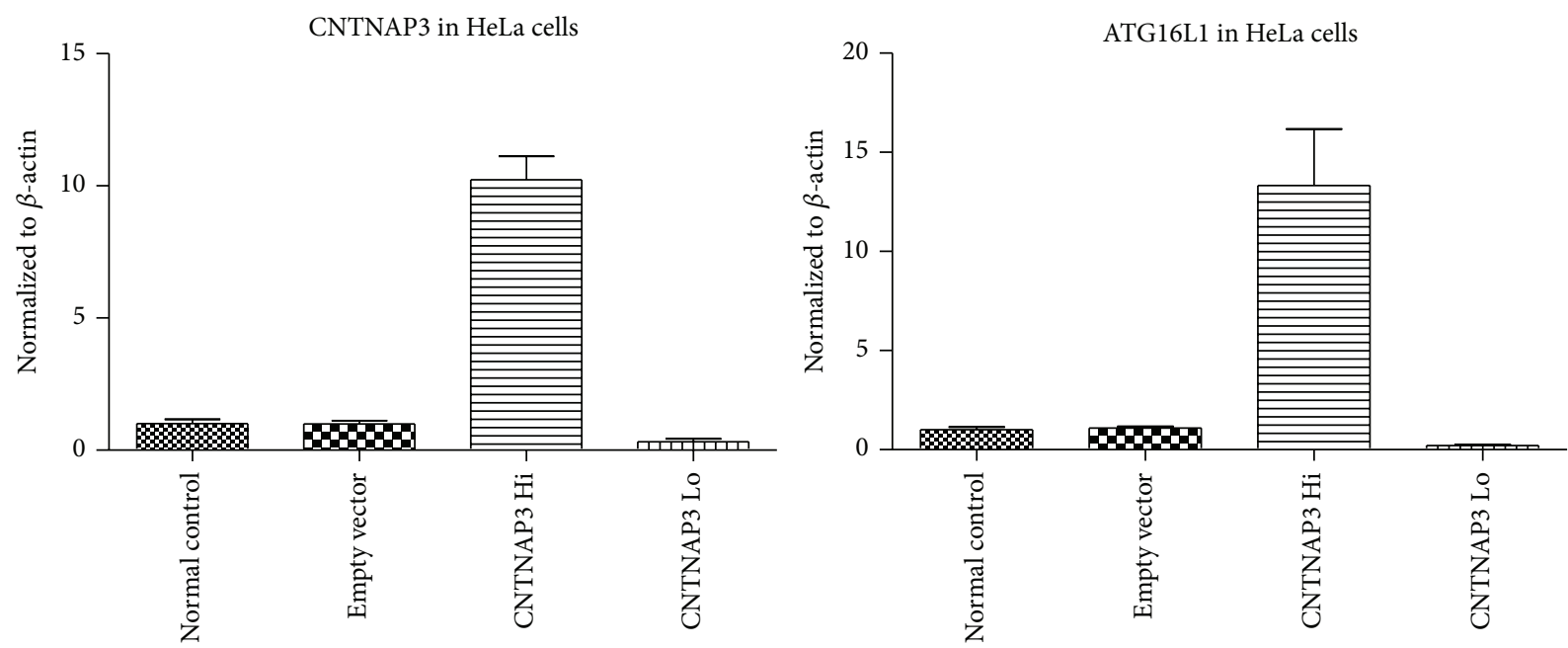

(a)
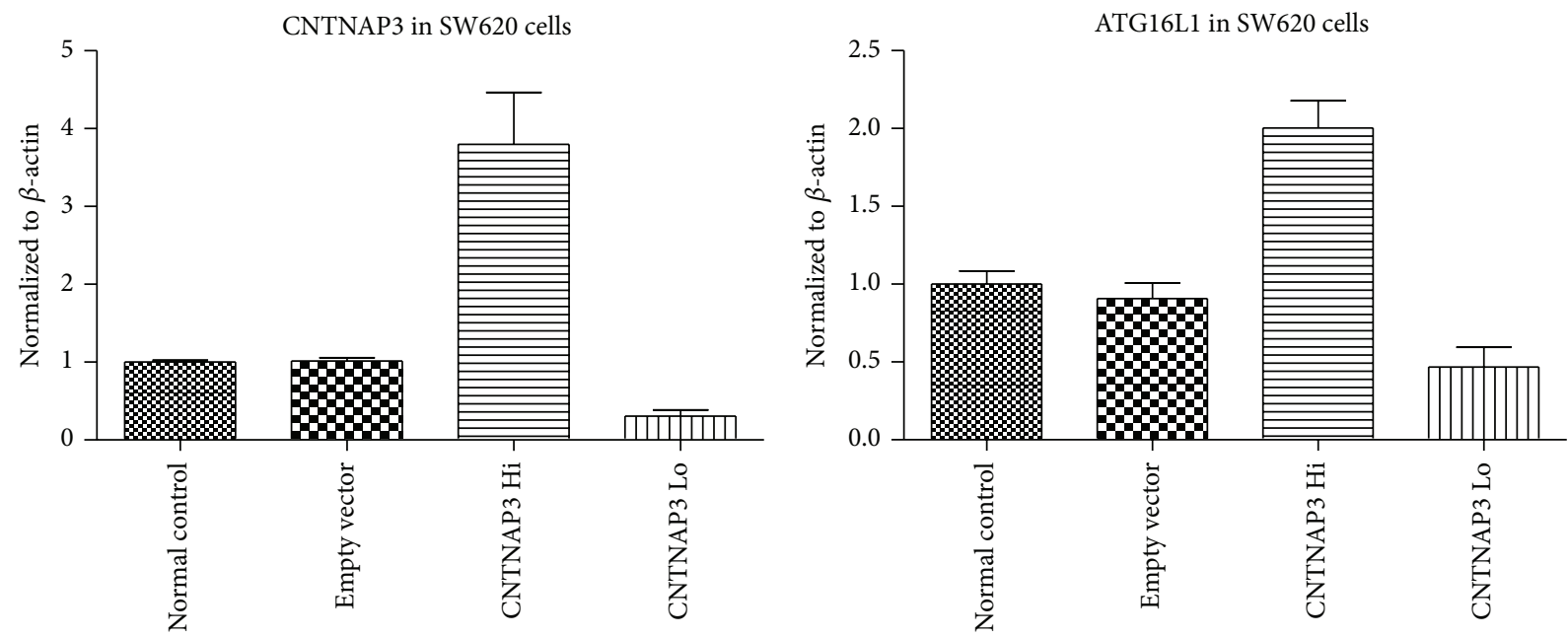

(b)

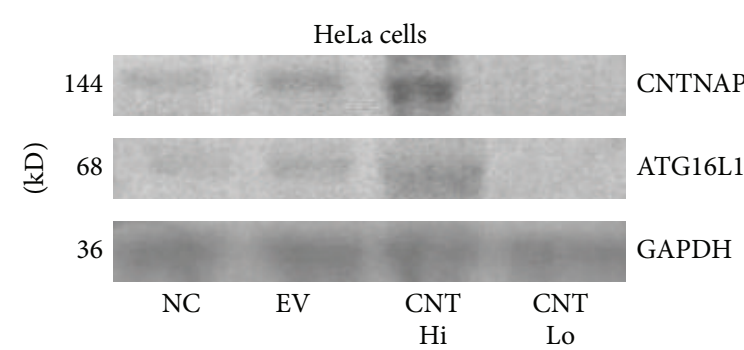

(c)

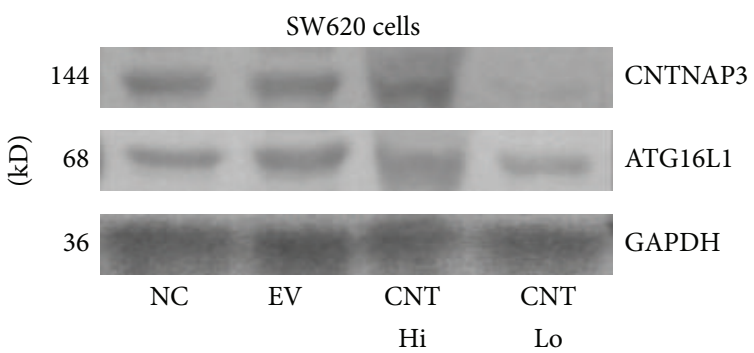

(d)

FiguRE 3: Level of ATG16L1 after CNTNAP3 manipulation. Seventy-two hours after transfection, ATG16L1 was lower in CNTNAP3 knockdown cells than that in cells transfected with empty vectors (HeLa, $P=0.013$ and SW620, $P=0.005$ ). (a and b). On the other hand, ATG16L1 was significantly higher in cells transfected with CNTNAP3 overexpression plasmid than that in cells transfected with empty vectors (HeLa, $P=0.001$ and SW620, $P=0.052$ ) (a and b). There was no significant difference between cells transfected with empty vectors and normal controls (HeLa, $P=0.630$ and SW620, $P=0.517$ ). Similarly, western blot indicated that ATG16L1 protein expression was significantly associated with the expression of CNTNAP3 manipulations. ATG16L1 protein expression significantly decreased and increased 72 hours after CNTNAP3 siRNA interference and CNTNAP3 overexpression, respectively (c and d). 

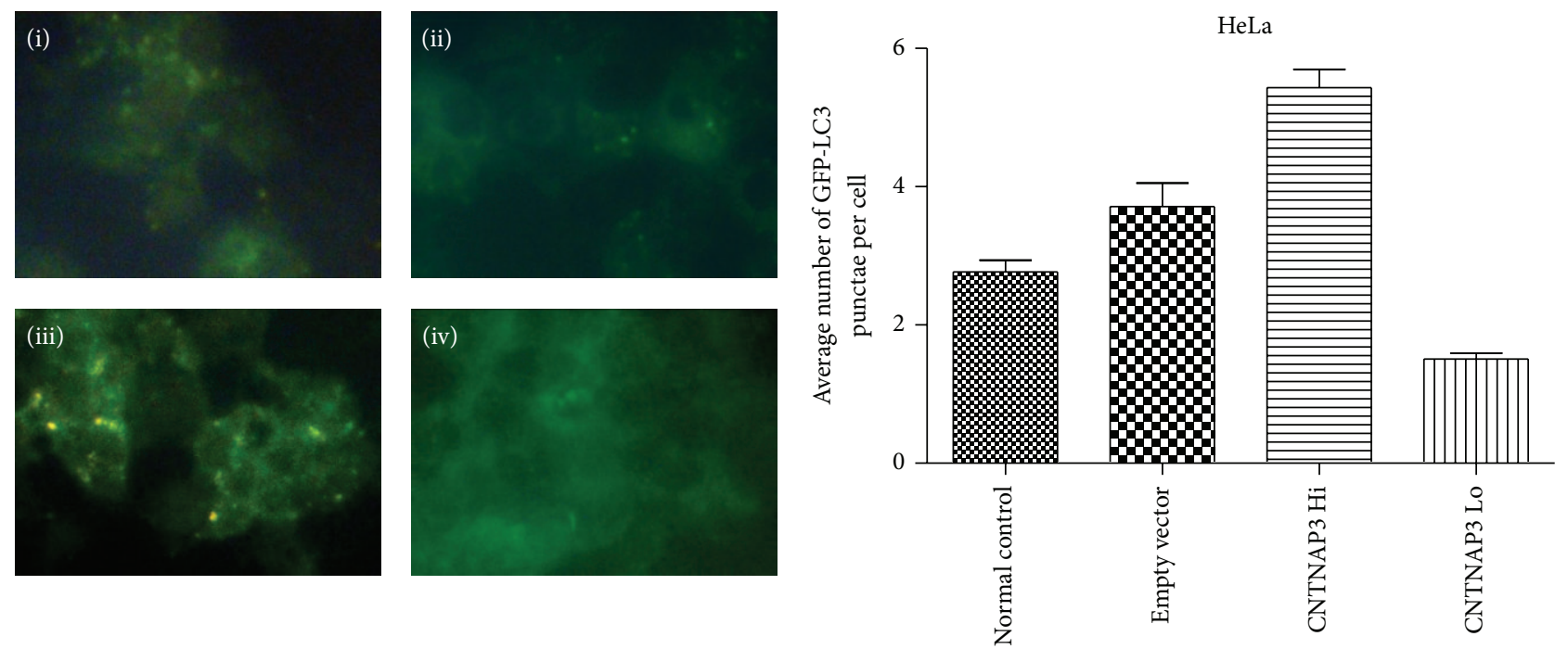

(a)
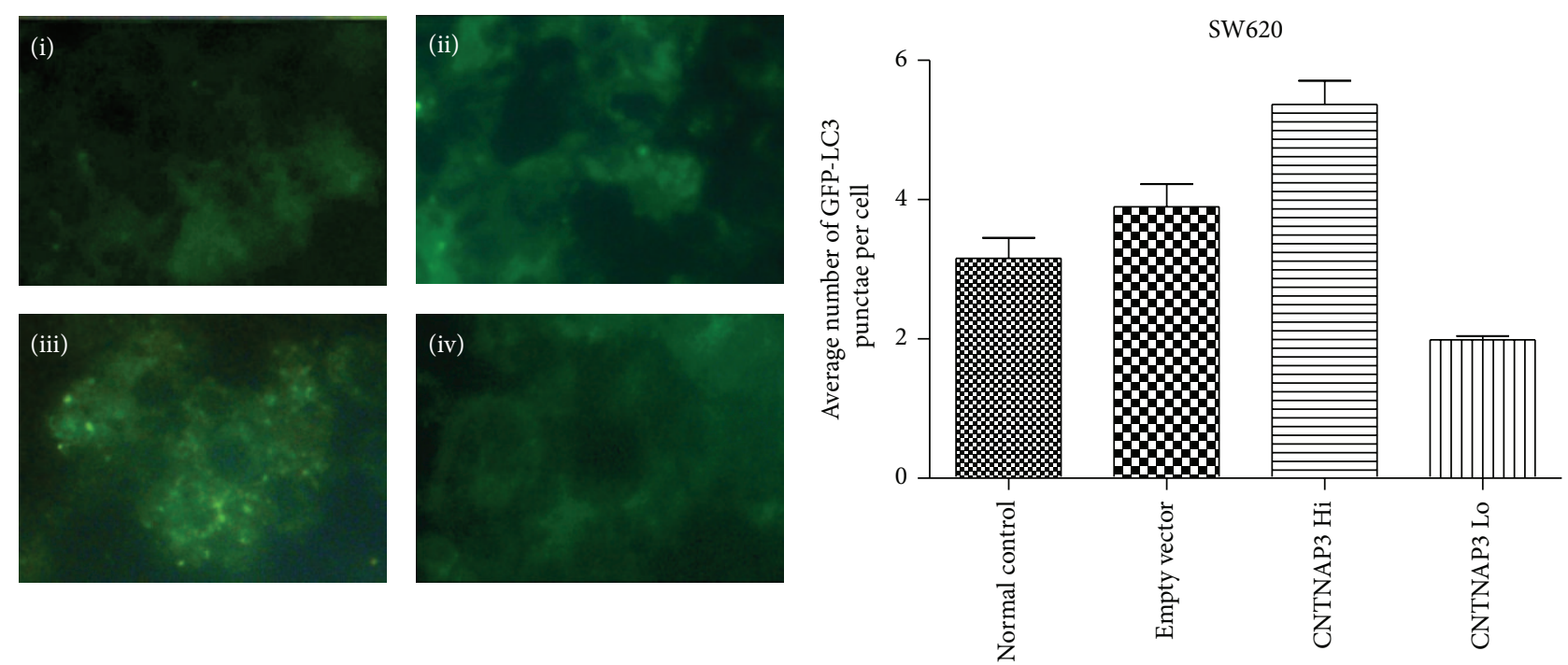

(b)

FIGURE 4: Autophagy phenomenon after CNTNAP3 manipulation. (a) In HeLa cells, the average number of GFP-LC3 punctuated dots per cell of CNTNAP3 overexpression group was $5.434 \pm 0.2658$, comparing to $1.507 \pm 0.08273$ in CNTNAP3 knockdown group and $3.714 \pm 0.3373$ in empty vector group. (b) In SW620 cells, the average number of GFP-LC3 punctuated dots per cell of CNTNAP3 overexpression group was $5.365 \pm 0.3412$, while it was $1.989 \pm 0.05458$ in CNTNAP3 knockdown group and $3.899 \pm 0.3226$ in empty vector group. It was shown that autophagy vacuoles significantly increased (HeLa, $P=0.161$ and SW620, $P=0.036)$ and decreased $(\mathrm{HeLa}, P=0.003$ and SW620, $P=0.004$ ) after CNTNAP3 overexpression and CNTNAP3 siRNA interference compared with cells transfected with empty vectors. ((i) Normal control; (ii) empty vector; (iii) CNTNAP3 overexpression; (iv) CNTNAP3 knockdown).

transfected with empty vectors and normal controls (HeLa, $P=0.630$ and SW620, $P=0.517)$.

Similarly, western blot also indicated that ATG16L1 protein expression was significantly associated with the expression of CNTNAP3 manipulations. ATG16L1 protein expression significantly decreased or increased 72 hours after CNTNAP3 was interfered by siRNA and CNTNAP3 overexpressed, respectively (Figures 3(c) and 3(d)).

3.5. CNTNAP3 Regulates Autophagy in HeLa and SW620 Cells. To determine whether autophagy can be regulated by
CNTNAP3, HeLa and SW620 cells were cotransfected with LC3-GFP plasmid and subsequently observed using fluorescent microscope 72 hours after CNTNAP3 modulation.

It was shown that autophagy vacuoles significantly increased (HeLa, $P=0.161$ and SW620, $P=0.036$ ) (Figures 4(a) (iii) and 4(b) (iii)) and decreased (HeLa, $P=0.003$ and SW620, $P=0.004$ ) (Figures 4(a) (iv) and 4(b) (iv)) after CNTNAP3 overexpression and siRNA interference compared with cells transfected with empty vectors (Figures 4(a) (ii) and 4(b) (ii)). No significant difference was found between empty vector group and normal control (HeLa, $P=0.066$ and 
SW620, $P=0.166)$. In HeLa cells, average number of GFPLC3 punctuated dots per cell of CNTNAP3 overexpression group was $5.434 \pm 0.2658$. It was $1.507 \pm 0.08273$ in CNTNAP3 knockdown group and $3.714 \pm 0.3373$ in empty vector group. In SW620 cells, the average number of GFP-LC3 punctuated dots per cell of CNTNAP3 overexpression group was $5.365 \pm$ 0.3412. It was $1.989 \pm 0.05458$ in CNTNAP3 knockdown group and $3.899 \pm 0.3226$ in empty vector group (Figures 4 (a) and $4(\mathrm{~b}))$.

\section{Discussion}

ATG16Ll is a classic gene that is associated with the development of Crohn's disease. In early research, Hampe et al. identified that ATG16L1 variant was associated with susceptibility to Crohn's disease [1]. And then a nonsynonymous SNP was identified to show association of a threonine-toalanine substitution (T300A) in ATG16L1. With ATG16L1 (T300A), the innate immune cells cannot be triggered by the specific microbial structures to form the autophagosomes. This phenomenon leads to subsequent bacterial persistence [18]. Interestingly, expression of ATG16L1 was independent of the ATG16L1 (T300A) genotype [1]. Fujita et al. demonstrated that ATG16L1 (T300A) mutant had little impact on canonical autophagy [19]. Although ATG16L1 polymorphisms can lead to subsequent bacterial persistence in the gut mucosa, 25\% of Caucasian populations with the risk allele do not have severe gut inflammation [18]. Therefore, ATG16L1 variants could not be the only cause of the disease. On the other hand, many patients with Crohn's disease do not have the risk allele ATG16L1 (T300A). In Italian [20], Brazilian [21], Moroccan [22], Japanese [11], and Korean [12] populations, ATG16L1 (T300A) was not found associated with CD. Although ATG16L1 (T300A) was found in Asian populations, the relationship of the variants and Asian Crohn's disease is still to be confirmed. In some other autoimmune diseases, ATG16L1 was also found upregulated in dendritic cells [23].

In our research, ATG16L1 expression in colonic mucosal samples from CD patients was found to be increased, and mRNA expression of ATG16L1 was correlated with CRP of patients. This phenomenon showed that ATG16L1 played an important role in the course of the disease, and the relationship between ATG16L1 and CNTNAP3 showed that CNTNAP3 might be of importance in autophagy process of Crohn's disease. As newly reported in gut related disease, CNTNAP3 is a distinct subgroup of the neurexins [13]. It was first found in nervous system, the function of CNTNAP3 remaining undiscovered. In our study, we found CNTNAP3 was highly expressed in colonic samples from Crohn's disease patients, and it might increase the autophagy process in cell models. Correlation between CNTNAP3 and ATG16L1 was significantly high. As most reports have confirmed, variants of ATG16L1 gene were related to autophagy deficiency. Contrary to expectation, overexpression of ATG16L1 did not definitely enhance the autophagy. Interestingly, abrupt increase of ATG16L1 could lead to reduced autophagy [24]. ATG16L1 was found to work together with ATG12 and ATG5 in the formation of autophagosomes [25], of which the process was dependent on the correct localization of ATG16L1 to sites of LC3-lipidation. In our research, there were no apparent autophagosomes forming disruption in any group. With the increase of CNTNAP3 and ATG16L1, autophagosomes increased in HeLa and SW620 cells subsequently.

Bacterial immunity was found disabled in autophagy deficiency related to mutations of ATG16L1 or NOD2 [26, 27]. In our research, autophagosomes increased without the stimulation of bacteria. It might be an adaptive reaction of cells under the overexpression of CNTNAP3. Necroptosis of cells might be another explanation. Necroptosis, a programmed cell death which is believed to link with the pathogenesis of IBD, shows the features of necrosis, including mitochondrial swelling and extensive vacuole formation [28]. Autophagy vacuoles are commonly seen during the process of necroptosis.

In our study, biopsies from CD patients showed a higher ATG16L1 mRNA level. ATG16L1 was well correlated with the CRP values in patients indicating that ATG16L1 might be a biomarker for the severity of the disease.

$\begin{array}{ll}\text { Abbreviations } & \\ \text { CD: } & \text { Crohn's disease } \\ \text { NOD2-ATG16L1: } & \begin{array}{l}\text { Nucleotide oligomerization } \\ \text { domain 2-autophagy genes } \\ \text { autophagy 16-like 1 }\end{array} \\ & \text { Contactin associated protein-like } \\ \text { CNTNAP3: } & 3 \\ \text { CRP: } & \text { C-reactive protein } \\ \text { GWAS: } & \text { Genome-wide association studies } \\ \text { ATG16L1: } & \text { Autophagy-related 16-like 1 } \\ \text { IRGM: } & \text { Immunity-related GTPase family } \\ & \text { M } \\ \text { NOD2: } & \text { Nucleotide oligomerization } \\ & \text { domain 2 } \\ \text { Neuroxin-IV/CNTNAP/ } & \text { NCP family } \\ \text { Paranodin: } & \text { Healthy controls } \\ \text { HC: } & \text { Erythrocyte sedimentation rate } \\ \text { ESR: } & \text { American Type Culture } \\ \text { ATCC: } & \text { Collection } \\ \text { DMEM: } & \text { Dulbecco's Modified Eagle } \\ \text { MFA: } & \text { Medium } \\ \text { BMI: } & \text { Monodansylcadaverine } \\ & \text { Paraformaldehyde } \\ \text { Body mass index. }\end{array}$

\section{Conflict of Interests}

The authors declare that they have no competing interests.

\section{Authors' Contribution}

Yu Qi Qiao and Mei Lan Huang contributed equally to this paper. 


\section{Acknowledgment}

This study is supported by the National Natural Science Foundation of China (Grants nos. 81000161, 81170362, 81200280, and 81370508).

\section{References}

[1] J. Hampe, A. Franke, P. Rosenstiel et al., "A genome-wide association scan of nonsynonymous SNPs identifies a susceptibility variant for Crohn disease in ATG16L1," Nature Genetics, vol. 39, no. 2, pp. 207-211, 2007.

[2] M. Parkes, J. C. Barrett, N. J. Prescott et al., "Sequence variants in the autophagy gene IRGM and multiple other replicating loci contribute to Crohn's disease susceptibility," Nature Genetics, vol. 39, no. 7, pp. 830-832, 2007.

[3] T. Saitoh, N. Fujita, M. H. Jang et al., "Loss of the autophagy protein Atg16L1 enhances endotoxin-induced IL-1 $\beta$ production," Nature, vol. 456, no. 7219, pp. 264-268, 2008.

[4] T. S. Plantinga, T. O. Crisan, M. Oosting et al., "Crohn's disease-associated ATG16L1 polymorphism modulates proinflammatory cytokine responses selectively upon activation of NOD2," Gut, vol. 60, no. 9, pp. 1229-1235, 2011.

[5] J. Harris, M. Hartman, C. Roche et al., "Autophagy controls ILlbeta secretion by targeting pro-IL-1beta for degradation," The Journal of Biological Chemistry, vol. 286, no. 11, pp. 9587-9597, 2011.

[6] A. Franke, D. P. McGovern, J. C. Barrett et al., "Genome-wide meta-analysis increases to 71 the number of confirmed Crohn's disease susceptibility loci," Nature Genetics, vol. 42, no. 12, pp. 1118-1125, 2010.

[7] J. C. Barrett, S. Hansoul, D. L. Nicolae et al., "Genome-wide association defines more than 30 distinct susceptibility loci for Crohn's disease," Nature Genetics, vol. 40, no. 8, pp. 955-962, 2008.

[8] R. Cooney, J. Baker, O. Brain et al., "NOD2 stimulation induces autophagy in dendritic cells influencing bacterial handling and antigen presentation," Nature Medicine, vol. 16, no. 1, pp. 90-97, 2010.

[9] L. H. Travassos, L. A. M. Carneiro, M. Ramjeet et al., "Nod1 and Nod2 direct autophagy by recruiting ATG16L1 to the plasma membrane at the site of bacterial entry," Nature Immunology, vol. 11, no. 1, pp. 55-62, 2010.

[10] L. Galluzzi, O. Kepp, L. Zitvogel, and G. Kroemer, "Bacterial invasion: linking autophagy and innate immunity," Current Biology, vol. 20, no. 3, pp. R106-R108, 2010.

[11] K. Yamazaki, Y. Onouchi, M. Takazoe, M. Kubo, Y. Nakamura, and A. Hata, "Association analysis of genetic variants in IL23R, ATG16L1 and 5p13.1 loci with Crohn's disease in Japanese patients," Journal of Human Genetics, vol. 52, no. 7, pp. 575-583, 2007.

[12] S.-K. Yang, M. Park, J. Lim et al., "Contribution of IL23R but not ATG16L1 to Crohn's disease susceptibility in Koreans," Inflammatory Bowel Diseases, vol. 15, no. 9, pp. 1385-1390, 2009.

[13] I. Spiegel, D. Salomon, B. Erne, N. Schaeren-Wiemers, and E. Peles, "Caspr3 and caspr4, two novel members of the caspr family are expressed in the nervous system and interact with PDZ domains," Molecular and Cellular Neuroscience, vol. 20, no. 2, pp. 283-297, 2002.

[14] K. J. Livak and T. D. Schmittgen, "Analysis of relative gene expression data using real-time quantitative PCR and the $2^{-\Delta \Delta C_{t}}$ method," Methods, vol. 25, no. 4, pp. 402-408, 2001.
[15] A. Biederbick, H. F. Kern, and H. P. Elsasser, "Monodansylcadaverine (MDC) is a specific in vivo marker for autophagic vacuoles," European Journal of Cell Biology, vol. 66, no. 1, pp. 314, 1995.

[16] R. A. González-Polo, P. Boya, A. L. Pauleau et al., “The apoptosis/autophagy paradox: autophagic vacuolization before apoptotic death," Journal of Cell Science, vol. 118, no. 14, pp. 30913102, 2005.

[17] N. Mizushima, T. Yoshimori, and B. Levine, "Methods in mammalian autophagy research," Cell, vol. 140, no. 3, pp. 313326, 2010.

[18] T. S. Plantinga, L. A. B. Joosten, and M. G. Netea, "ATG16L1 polymorphisms are associated with NOD2-induced hyperinflammation," Autophagy, vol. 7, no. 9, pp. 1074-1075, 2011.

[19] N. Fujita, T. Saitoh, S. Kageyama, S. Akira, T. Noda, and T. Yoshimori, "Differential involvement of Atg16L1 in Crohn disease and canonical autophagy: analysis of the organization of the Atg16L1 complex in fibroblasts," The Journal of Biological Chemistry, vol. 284, no. 47, pp. 32602-32609, 2009.

[20] C. Perricone, P. Borgiani, S. Romano et al., "ATG16L1 Ala197Thr is not associated with susceptibility to Crohn's disease or with phenotype in an Italian population," Gastroenterology, vol. 134, no. 1, pp. 368-370, 2008.

[21] M. L. Baptista, H. Amarante, G. Picheth et al., "CARD15 and IL23R influences Crohn's disease susceptibility but not disease phenotype in a Brazilian population," Inflammatory Bowel Diseases, vol. 14, no. 5, pp. 674-679, 2008.

[22] N. Serbati, N. Senhaji, B. Diakite, W. Badre, and S. Nadifi, "IL23R and ATG16L1 variants in Moroccan patients with inflammatory bowel disease," BMC Research Notes, vol. 7, no. 1, article 570, 2014.

[23] M. H. Wenink, K. C. M. Santegoets, J. Butcher et al., "Impaired dendritic cell proinflammatory cytokine production in psoriatic arthritis," Arthritis \& Rheumatism, vol. 63, no. 11, pp. 3313-3322, 2011.

[24] N. Fujita, T. Itoh, H. Omori, M. Fukuda, T. Noda, and T. Yoshimori, "The Atg16L complex specifies the site of LC3 lipidation for membrane biogenesis in autophagy," Molecular Biology of the Cell, vol. 19, no. 5, pp. 2092-2100, 2008.

[25] N. Mizushima, T. Yoshimori, and Y. Ohsumi, "The role of atg proteins in autophagosome formation," Annual Review of Cell and Developmental Biology, vol. 27, pp. 107-132, 2011.

[26] C. R. Homer, A. L. Richmond, N. A. Rebert, J. Achkar, and C. McDonald, "ATG16L1 and NOD2 interact in an autophagydependent antibacterial pathway implicated in Crohn's disease pathogenesis," Gastroenterology, vol. 139, no. 5, pp. 1630.e21641.e2, 2010.

[27] P. Kuballa, A. Huett, J. D. Rioux, M. J. Daly, and R. J. Xavier, "Impaired autophagy of an intracellular pathogen induced by a Crohn's disease associated ATG16L1 variant," PLoS ONE, vol. 3, no. 10, Article ID e3391, 2008.

[28] C. Günther, H. Neumann, M. F. Neurath, and C. Becker, "Apoptosis, necrosis and necroptosis: cell death regulation in the intestinal epithelium," Gut, vol. 62, no. 7, pp. 1062-1071, 2013. 


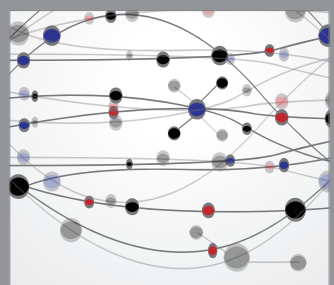

The Scientific World Journal
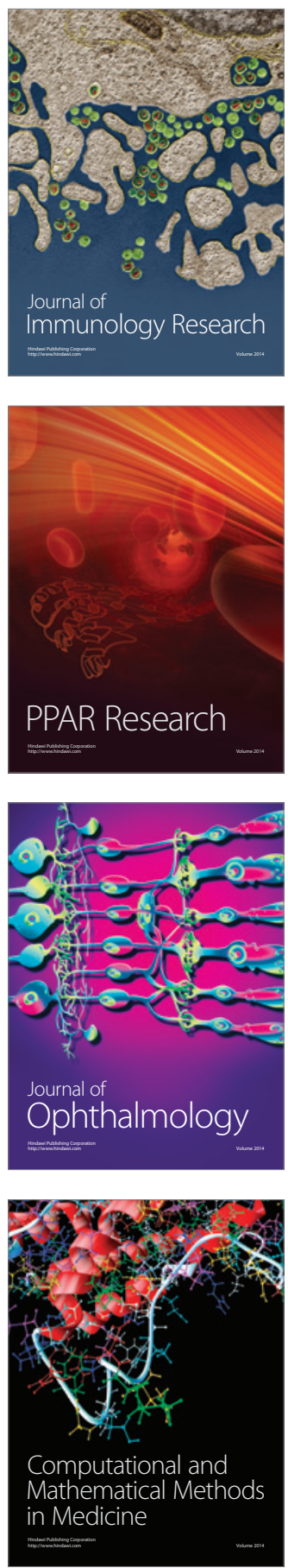

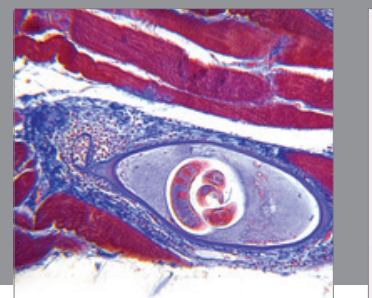

Gastroenterology

Research and Practice
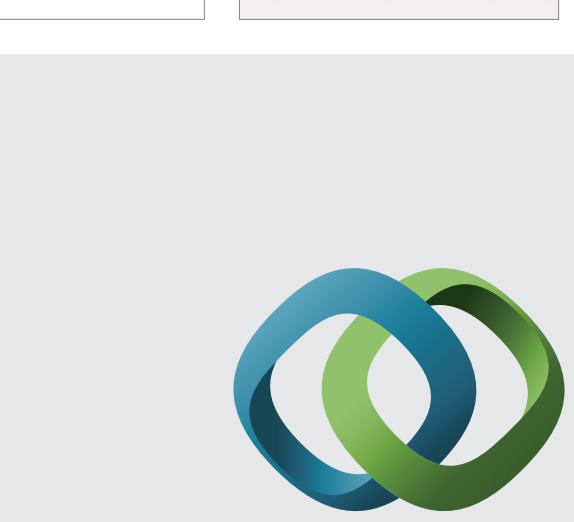

\section{Hindawi}

Submit your manuscripts at

http://www.hindawi.com
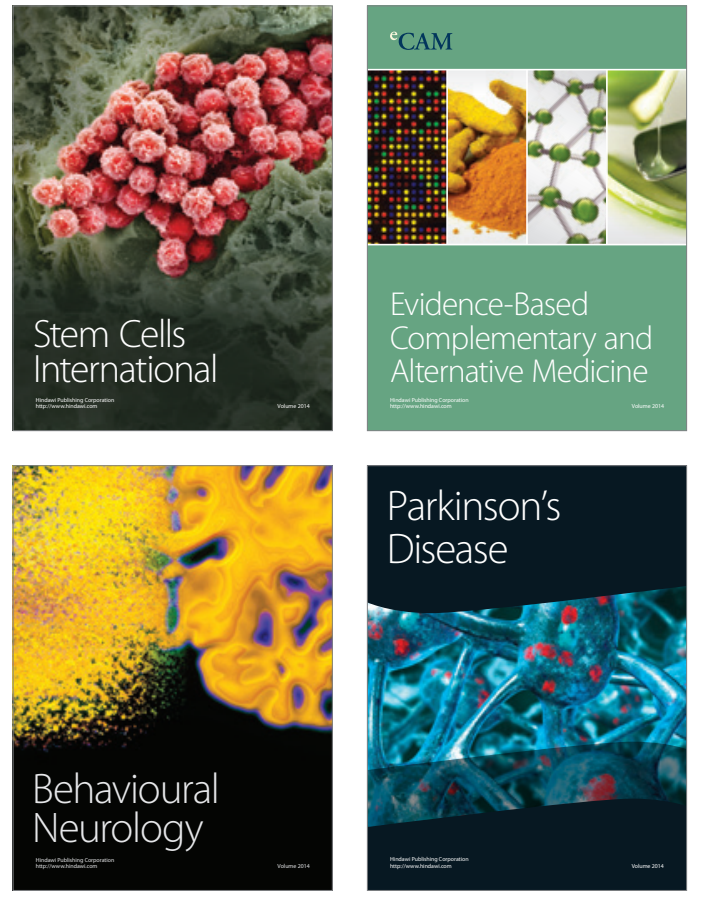
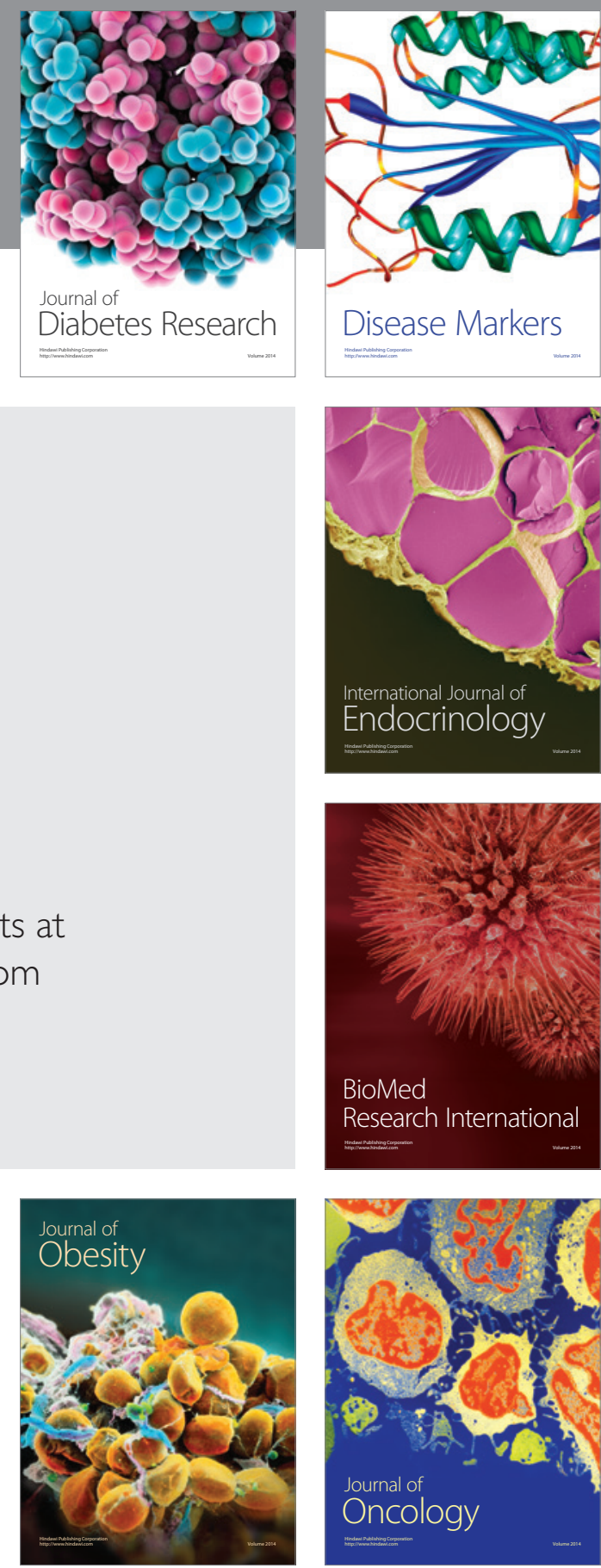

Disease Markers
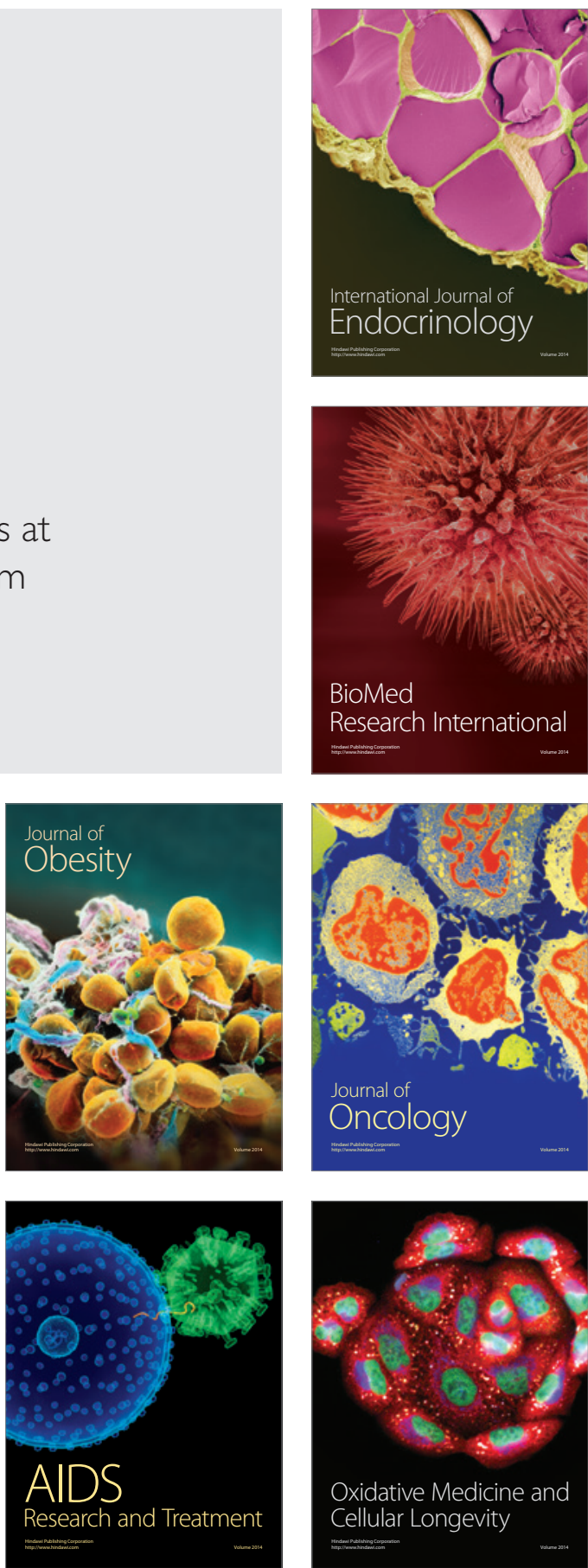